\title{
Physical Vacuum and Cosmic Coincidence Problem
}

\author{
Arthur D. Chernin \\ Sternberg Astronomical Institute, Moscow University, Moscow, 119899, Russia, \\ Astronomy Division, FIN-90014 University of Oulu, Finland, \\ and Tuorla Observatory, University of Turku, Piikkiö, FIN-21500, Finland
}

\begin{abstract}
A framework is suggested in which the energy integrals of the Friedmann cosmology are identified as genuine time-independent physical characteristics for both vacuum and non-vacuum forms of cosmic energy. The integrals are found to be numerically coincident within two orders of magnitude. It is assumed that this coincidence reveals a symmetry that relates vacuum to non-vacuum forms of cosmic energy at fundamental level. The symmetry shows the cosmic coincidence problem and the naturalness problem as two inter-related aspects of a more general problem: Why are the energy integrals numerically coincident and equal to $\sim 10^{60} M_{P l}^{-1}$ ? A simple kinetics model of cosmological freeze out is used to examine how - at least, in principle - the electroweak scale physics might explain the nature of the symmetry between vacuum and non-vacuum cosmic energies and determine the value of the energy integrals in terms of the fundamental energy scales.
\end{abstract}

Keywords: Cosmology; Dark matter

PACS numbers: 04.70.Dy; 04.25.Dm; 04.60-m; 95.35.+d; 98.80.Cq 


\section{Introduction}

The four major forms of energy in current cosmological models are the acceleration (A) energy which is vacuum and/or a rolling scalar quintessential field, cold dark (D) matter, baryons (B) and ultra-relativistic (R) energy which includes cosmic microwave background $(\mathrm{CMB})$ photons and other possible light or massless particles (neutrinos, gravitons, etc.) of cosmological origin. The first of these forms of cosmic energy has recently been discovered in the observations of high-redshift supernovae (Ries et al. 1998, Perlmutter et al. 1999), and this has been also confirmed by evidence from the cosmic age, the large-scale structure, the CMB anisotropy in combination with cluster dynamics and galaxy velocity field, etc. (see Cohn 1998, Carol 2000, Bahcall et al. 2000 and references therein).

The best fit concordant with the bulk of all observational evidence today is provided by the following figures for the densities of these forms of energy (see the references above):

$$
\Omega_{A}=0.7 \pm 0.1, \quad \Omega_{D}=0.3 \pm 0.1, \quad \Omega_{B}=0.02 \pm 0.01, \quad \Omega_{R}=0.6 \alpha \times 10^{-4}
$$

where $1<\alpha<30-100$ is a dimensionless constant factor that accounts for non-CMB contributions to the relativistic energy. With the Hubble constant $h_{100}=0.65 \pm 0.15$, the figures lead to either an open cosmological model or a spatially flat model.

The density of A-energy, $\rho_{A} \sim 10^{-123} M_{P l}^{4}$ (in the units in which $c=k=\hbar=$ $1 ; M_{P l}=1.2 \times 10^{19} \mathrm{GeV}$ is the Planck mass), conflicts drastically with simple field theoretic expectations, if this density is treated as the density of physical vacuum. Symmetry arguments may explain why the vacuum density should be either zero or Planckian, but there is no explanation from the 'first principles' for a non-zero, tiny and positive vacuum density. This is the well-known 'naturalness problem in theoretical physics' (Weinberg 1989).

On the other hand, the density of acceleration matter is near-coincident with the density of dark matter and also with the densities of the two other forms of non-vacuum energy, as is seen from Eq.(1); this is another problem, known as the 'cosmic coincidence problem', that has been recognized as a severe challenge to current cosmological concepts 
(Wang et al. 2000).

One possible approach to both problems would be to assume that the acceleration of the expansion is produced by non-vacuum energy which has negative pressure and negative effective gravitating density (Peebles and Ratra 1988, Frieman and Waga 1998, Caldwwell and Steinhardt 1998, Caldwell et al. 1998, Zlatev et al. 1999). This energy form is called quintessence; it can naturally be realized in some scalar field models in which the field depends on time, and so the density of quintessence is diluted with the expansion. If so, the densities involved in the observed cosmic coincidence are all diluted, which makes them seemingly more similar to each other. Quintessence density might temporarily or even always be comparable to the density of dark matter. Although such an idea may make the closeness of all cosmic densities natural, it does not explain the coincidence that the quintessence field becomes settled with a finite energy density comparable to the matter energy density just now (see, for instance, Arkani-Hamed et al. 2000).

In the present paper, a framework is suggested that is alternative to the idea of quintessence. The cosmic acceleration is understood below as a dynamical effect of physical vacuum with constant density $\rho_{V}=\rho_{A}$ in any reference frame. In this framework, the cosmic coincidence problem and the naturalness problem are treated as two basic aspects of a more fundamental problem in theoretical physics and cosmology. This new problem can be formulated in terms of energy integrals of the Friedmann cosmology.

In Secs.2,3 it is demonstrated that the energy integrals are constant genuine physical characteristics of both vacuum and non-vacuum energy forms; on this basis, the formulation of the problem is given in Sec.4; in Sec.5, it is argued that the electroweak scale physics can be the major mediator in the interplay between vacuum and non-vacuum energy forms, and a kinetics model that describes this process at $\mathrm{TeV}$ temperatures in early Universe is studied in Sec.6; a brief summary is given in Sec.7. 


\section{$2 \quad$ Energy integrals}

The constant energy integrals enter the general Friedmann solution for the four cosmic energies mentioned above:

$$
\int d a\left(A_{V}^{-2} a^{2}+2 A_{D} a^{-1}+2 A_{B} a^{-1}+A_{R}^{2} a^{-2}-K\right)^{-1 / 2}=t,
$$

were $a(t)$ is the curvature radius and/or a scale factor of the model, $K=1,0,-1$, accordingly to the sign of the spatial curvature. Constants $A$ come from the Friedmann 'thermodynamic' equation which is equivalent to energy and entropy conservation relation in a co-moving volume during the process of adiabatic expansion:

$$
A=\left[\left(\frac{1+3 w}{2}\right)^{2} \kappa \rho a^{3(1+w)}\right]^{\frac{1}{1+3 w}} .
$$

Here $w=p / \rho$ is the pressure-to-density ratio for a given energy form; $w=-1,0,0,1 / 3$ for vacuum, dark matter, baryons and radiation, respectively; $\kappa=8 \pi G / 3=(8 \pi / 3) M_{P l}^{-2}$. The interpretation of the constants $A_{D}, A_{B}, A_{R}$ is quite obvious in Eq.(3) for non-vacuum energies: they express the conservation of the number of particles in the co-moving volume. It is most interesting that the constant for vacuum $A_{V}$ is also given by the same general relation of Eq.(3), while the interpretation in terms of particles does not work in this later case.

In an explicit form, the solution of Eq.(3) may be written separately for the earlier epoch $\left(z>z_{V} \simeq 1\right)$, when the non-vacuum energies dominate and for the later vacuum domination epoch $\left(z<z_{V}\right)$. For a spatially flat model at $z>z_{V}$ one has (Chernin 1965)

$$
a(\eta)=A_{M} \eta^{2}+A_{R} \eta, \quad t(\eta)=\frac{1}{3} A_{M} \eta^{3}+\frac{1}{2} A_{R} \eta^{2} .
$$

Here $\eta$ is conformal time, and $A_{M}, A_{R}$ are given by Eq.(3) with $\rho=\rho_{M}=\rho_{D}+\rho_{B}, w=0$ and $\rho=\rho_{R}, w=1 / 3$, respectively.

For the vacuum domination epoch, one has a well-known solution that describes accelerating expansion controlled by vacuum only:

$$
a(t)=A_{V} f(t), \quad f(t)=\sinh \left(t / A_{V}\right), \quad \exp \left(t / A_{V}\right), \quad \cosh \left(t / A_{V}\right)
$$

for open, spatially flat and close models, respectively. The present-day $\left(t=t_{0} \simeq 15 \pm 2\right.$ Gyr) value of $a(t)$ is estimated with this solution and the observed Hubble constant; one has approximately: $a\left(t_{0}\right) \sim A_{V}$ for all the three models. 
Eqs.(2-5) suggest a framework in which the time-independent integrals for all the four energy forms are considered as basic cosmological quantities.

Indeed, the energy integrals given by Eq.(3) are genuine constant characteristics for the respective forms of energy during all the time when each of the energies exists (and not only at the epoch when a given energy dominates - see the solutions of Eqs.2,4,5 above). Each of the energies is represented by its corresponding integral independently from other components. Being constants of integration, the integrals are completely arbitrary, in the sense that the Friedmann equations provide no limitations on them, except for trivial ones. From the view point of physics, the integrals are determined by 'initial conditions' at the epoch of the origin of the forms of energy in the early Universe; at that time, each of the energies acquires its own integral $A$ as a genuine quantitative characteristic, which is then kept constant in time.

\section{Evaluation of energy integrals}

The numerical estimation of the integrals can be made with the figures of Eq.(1) and with the use of Eqs. $(3,5)$ :

$$
\begin{gathered}
A_{V}=\left(\kappa \rho_{A}\right)^{-1 / 2} \sim 10^{42} \mathrm{GeV}^{-1} \sim 10^{61} \mathrm{M}_{P l}^{-1}, \\
A_{D}=\frac{1}{4} \kappa \rho_{D} a^{3} \sim 10^{41} \mathrm{GeV}^{-1} \sim 10^{60} \mathrm{M}_{P l}^{-1}, \\
A_{B}=\frac{1}{4} \kappa \rho_{B} a^{3} \sim 10^{40} \mathrm{GeV}^{-1} \sim 10^{59} \mathrm{M}_{P l}^{-1}, \\
A_{R}=\left(\kappa \rho_{R}\right)^{1 / 2} a^{2} \simeq 10^{40} \mathrm{GeV}^{-1} \sim 10^{59} \mathrm{M}_{P l}^{-1} .
\end{gathered}
$$

The integrals (that have the dimension of the length) are evaluated for the open model with $a\left(t_{0}\right)$ given by Eq.(5). It is easy to see that the evaluation for the flat (with the scale factor normalized as it is indicated above in Eq.(5)) and close models gives rise

to similar results, on the order of magnitude. In the estimation of $A_{R}$, a conservative value $\alpha=1$ is adopted which takes into account the CMB photons only.

As one sees, the integrals have proven to be close to each other within two orders of magnitude, and this result may be summarized in a compact formula:

$$
A \sim 10^{60 \pm 1} M_{P l}^{-1}
$$


Note that the analysis may easily be extended to include quintessence alongside with or instead of vacuum. For instance, according to Eq.(3), quintessence with $w=-2 / 3$ is characterized by $A_{Q} \sim 10^{61} M_{P l}^{-1}$, if it is estimated with $\rho_{q}=\rho_{A}$ from Eq.(1). A special case is the integral for non-accelerating energy with $w=-1 / 3$ which is not contained in Eq.(3); using directly the corresponding solution of the Friedmann equations, one finds: $A_{-1 / 3}=A_{V}$, if $\rho_{-1 / 3}=\rho_{A}$.

\section{Symmetry of cosmic energy forms}

The integrals of Eqs. $(3,10)$ exist in the Universe since the epoch at which the four major forms of energy came to existence themselves, e.g. at least after $t \sim 1 \mathrm{sec}$, and will exist

until the decay of the protons at $t \geq 10^{31-32}$ yrs or the decay of the particles of dark matter. In the beginning of this time interval, the vacuum density is about forty orders of magnitude less than the density of R-energy that dominates at that epoch; but the numbers $A_{A}$ and $A_{R}$ are as close at $t \sim 1 \mathrm{sec}$ as they are in Eqs.(6,9). In the future, the scale factor will change in orders of of orders (!) of magnitude during the life-time of proton, and so the densities of D-, B-, and R-energies, as well as their ratios to the vacuum density, will change enormously. But the four constant numbers of Eq.(6-10) will remain the same keeping their near-coincidence for all this future time.

The empirical analysis of Secs. 2,3 has led to a novel version of the cosmic coincidences that appear now as a tetramerous coincidence of time-independent constant numbers - in contrast to the epoch-dependent (and therefore temporal, and accidental, in this sense) coincidence of densities. It is clear that the densities are coincident at the present epoch because this is the epoch of the transition from the decelerated matter dominated expansion to the accelerated vacuum domination expansion. Indeed, since the relation of Eq.10 exists from the early state of the Universe, the densities must be coincident just now, because $a(t) \sim A_{V}$ presently. Another question is why do we happen to live in a transition epoch; this is among the matters that may be discussed with antropic principle (see,for instance, Weinberg 1987).

The coincidence of the four integrals reveals a new type of basic regularities which are 
conserved in the evolving Universe. Since none of the energy forms looks preferable, in terms of the energy integrals of Eq.10, the regularity has a character of a symmetry that relates vacuum to non-vacuum forms of cosmic energy. The symmetry is not perfect, and its accuracy is within a few percent, on logarithmic scale, according to Eqs.(6-10).

The symmetry of two forms of energy, namely B- and R-energies, in terms of energy integrals, was first recognized soon after the discovery of the CMB (Chernin 1968). The significance of this B-R symmetry is obvious from the fact that the relation $A_{B} \sim$ $A_{R} \sim 10^{59} M_{P l}^{-1}$ enables alone to quantify such important things as the baryon-antibaryon asymmetry of the Universe, the entropy per baryon, the light-element production in the nucleosynthesis, the epoch of hydrogen recombination, the present-day temperature of CMB, etc.

In the framework of the symmetry extended to the all vacuum and non-vacuum cosmic energies, the genuine constant characteristic of physical vacuum $A_{V}$ finds its natural non-vacuum counterparts which are also constant in time. Since all the forms of cosmic energy are treated in a unified manner and on the common ground of the energy integrals, the naturalness problem looses its uniqueness and finds its place in this broader cosmological framework. This way the naturalness problem together with the cosmic coincidence problem are transformed into a more general problem in fundamental physics and cosmology: Why is $A_{V} \sim A_{D} \sim A_{B} \sim A_{R} \sim 10^{60} M_{P l}^{-1}$ ?

\section{$5 \quad$ Vacuum and electroweak scale physics}

In any fundamental unified theory, the value of the vacuum density would have to be calculable. Alongside with this, the symmetry of energy forms described by Eq.10 would have to be explained. This seems to be a rather remote goal. What could seemingly be done now is to try, first, to identify a clue physical factor that determines both the symmetry and the value of the vacuum density.

One possible way to do this is to assume that the vacuum density is completely due to zero oscillations of the quantum fields, as it was suggested not once since the 1930-s (see, for instance, Dolgov et al. 1988). If so, the vacuum density is given by an integral 
over all frequencies of the zero oscillations, - but this gives infinite value for the density. One may cut off the range of the frequencies, introducing a maximal frequency $\omega_{V}$; then the integral takes a form: $\rho_{V} \sim \omega_{V}^{4}$ (as it can be seen, for instance, from dimension considerations). With the observed vacuum density, one finds from this that $\omega_{V} \sim$ $10^{-31} M_{P l}$. Then one may put this maximal frequency into the cosmological context and compare it with the rate of cosmological expansion in the early Universe $1 / t \sim(G \rho)^{1 / 2}$; here $\rho \sim \rho_{R} \sim T^{4}$, and $T$ is the temperature of radiation which dominates at that times. From this one finds that $\omega_{V} \sim 1 / t$ at the epoch, when $T \sim 10^{-16} M_{P l} \simeq 1 \mathrm{TeV}$. The last value is close to the electroweak energy scale $M_{E W}$, and so $\omega_{V} \sim M_{E W}^{2} / M_{P l}$.

With this maximal frequency, the vacuum density is

$$
\rho_{V} \sim \omega_{V}^{4} \sim\left(M_{E W} / M_{P l}\right)^{8} M_{P l}^{4}
$$

These considerations suggest that the electroweak scale physics might be behind the observed density of cosmic vacuum. This physics may also be the major mediator between vacuum and non-vacuum forms of cosmic energies in the processes that might develop in the early Universe at the epoch of $\mathrm{TeV}$ temperatures.

Note that the relation of Eq.(11) was obtained also in a field-theoretic model (ArkaniHamed 2000, - unfortunately, under some arbitrary additional assumptions).

\section{$6 \quad$ A model}

Having in mind the central part that may be played by the electroweak scale physics at the epoch of $\mathrm{TeV}$ temperatures, one may try to examine, if this physics could determine also the symmetry between vacuum and non-vacuum energies. For this purposes, a kinetics model can be used, in which non-relativistic dark matter is considered as thermal relic of early cosmic evolution. This possibility has been widely discussed (see, for instance, the books by Zeldovich and Novikov 1983, Dolgov et al. 1988, Kolb and Turner 1990, and a recent work by Arkani-Hamed et al. 2000). The model below is incomplete: baryonic energy is not included in it, and so baryogenesis at $\mathrm{TeV}$ temperatures must be studied separately, but, perhaps, not independently of the model. As for vacuum, dark 
matter and radiation, they will be represented in the model by the corresponding energy integrals.

For stable (or long-living) particles of the mass $m$, the abundance freezes out when the temperature falls below the mass $m$ and the expansion rate $1 / t$ wins over the annihilation rate, $\sigma n$, where the annihilation cross-section $\sigma \sim m^{-2}$. So that at that moment the particle density is

$$
n \sim 1 /(\sigma t) \sim m^{2}\left(G \rho_{R}\right) .
$$

Using Eq.(3) for $A_{D}$ and $A_{R}$ and putting $\rho_{D} \sim m n$, one finds:

$$
A_{D} \sim a(t) m^{3} M_{P l}^{-2} A_{R}
$$

One also has at that moment $\rho_{R} \sim m^{4}$, and because of this

$$
A_{R} \sim a(t)^{2} m^{2} M_{P l}^{-1}
$$

where $a(t) \sim A_{V}(1+z)^{-1}$, and $z$ is the redshift at the freeze-out epoch; in this way, the vacuum integral comes to the model.

To specify the underlying fundamental physics, one can refer to a special significance of the electroweak scale physics, as it was mentioned above, and assume that only two fundamental energy scales are involved in the process, namely $M_{E W}$ and $M_{P l}$. If so, it is natural to identify the mass $m$ with the electroweak energy scale $M_{E W}$. Then, one may use the relation for the vacuum density in terms of the two fundamental energy scales, $M_{E W}$ and $M_{P l}$, as given by Eq.(11). With this density, the vacuum integral is

$$
A_{V} \sim\left(M_{P l} / M_{E W}\right)^{4} M_{P l}^{-1}
$$

Arguing along this line, one may expect that the redshift $z$ at the freeze-out epoch may be a simple combination of the same two mass scales:

$$
z \sim M_{P l} / M_{E W}
$$

Now the kinetics model is described by a system of four algebraic Eqs.(13-16) (with $\left.m=M_{E W}\right)$ for the four numbers $A_{M}, A_{R}, A_{V}, z$. The solution of the system is:

$$
A_{M} \sim A_{R} \sim A_{V} \sim\left(M_{P l} / M_{E W}\right)^{4} M_{P l}^{-1}
$$


Thus the coincidence of the three energy integrals appears as a direct result of the freeze-out process mediated by the electroweak scale physics. This physics determines also the value of the integrals.

Following Arkani-Hamed (2000) and another recent work by Kawasaki et al. (2000), one may introduce the gravitational scale $M_{G}$, or the reduced Planck scale $m_{P l}$, instead of the standard Planck scale: $M_{G} \simeq m_{P l} \simeq g M_{P l}$, where $g \simeq 0.1-0.3$. The dimensionless factor $g$ accounts for the fact that the gravity constant $G$ enters the exact cosmology relations in combinations like $8 \pi G / 3,6 \pi G$, or $32 \pi G / 3$. Similarly, a few dimensionless factors, like the effective number of degrees of freedom, etc., may also be included in the model - see again the books mentioned above. One gets finally:

$$
A \sim g^{4}\left(M_{P l} / M_{E W}\right)^{4} M_{P l}^{-1} \sim 10^{61 \pm 1} M_{P l}^{-1}, \quad w=[-1,0,1 / 3] .
$$

A quantitative agreement with the empirical result of Eq.(10) looks satisfactory here.

With $A_{V}$ of Eq.(15), one finds that the vacuum density is $\rho_{A} \sim g^{8}\left(M_{P l} / M_{E W}\right)^{8} M_{P l}^{4} \sim$ $10^{-122 \pm 2} M_{P l}^{4}$.

The numerical value of the redshift in Eq. (16) $z \sim g M_{P l} / M_{E W} \sim 10^{15}$, and so the temperature at the freeze-out is $T \sim 1 \mathrm{TeV} \sim M_{E W}$, which reflects once again the central role of the electroweak energy scale in the model above.

Thus, the model shows how - at least, in principle - the problem of the symmetry of cosmic energy forms can be discussed in terms of basic physics. The model gives an example of a possible (while partial and incomplete yet) solution to this problem.

\section{Conclusions}

The results of the discussion above may be summarized as follows:

1. A framework is suggested in which the energy integrals of the Friedmann cosmology equations (see Eqs.2,3) are identified as genuine time-independent physical characteristics for both vacuum and non-vacuum forms of cosmic energy.

2. The empirical analysis of the observational data on the four major energy forms leads to the conclusion that the corresponding energy integrals are numerically coincident within two orders of magnitude (Eqs.6-10). 
3. It is argued that the coincidence of the energy integrals found at the empirical level reveals a symmetry that relates vacuum to non-vacuum forms of cosmic energy at fundamental level.

4. The symmetry shows the cosmic coincidence problem and the naturalness problem as two inter-related aspects of a more general problem: Why are the energy integrals numerically coincident and equal to $\sim 10^{60} M_{P l}^{-1}$ ?

5. A special significance of the electroweak scale physics is demonstrated for this general problem.

6. Under the assumption that dark matter is a thermal relic of the early Universe, a simple kinetics model of cosmological freeze out is used to demonstrate how - at least, in principle - the electroweak scale physics might explain the nature of the symmetry between vacuum and non-vacuum cosmic energies and determine the value of the energy integrals in terms of the fundamental energy scales.

The work was partly supported by the grant of the Academy of Finland 'Galaxy streams and dark matter structures'.

\section{References}

Arkani-Hamed, N., Hall, L.J., Kolda, Ch., H. Murayama, H., 2000. Phys. Rev. Lett. 85,4434 .

Bahcall, N., Ostriker, J., Perlmutter, S., and Steinhard, P., 1999. Science 284, 1481.

Caldwell, R.R., and Steinhardt, P.J., 1998. Phys.Rev. D 57, 6057.

Caldwell, R.R., Davé, R., and Steinhardt, P.J., 1998. Phys.Rev. Lett. 80, 1582.

Carol, S., 2000. astro-ph/0004075.

Chernin, A.D., 1965. Sov. Astron. 42, 1124.

Chernin, A.D., 1968. Nature (London) 220, 250.

Cohn, J., 1998. astro-ph/9807128.

Dolgov, A.D., Zeldovich, Ya.B., Sazhin, M.V., 1988. Cosmology of the Early Universe. (In Russian; Moscow Univ. Press, Moscow.

Frieman, J.A., Waga, I., 1998. Phys. Rev. D 57, 4642. 
Kawasaki, M., Yamaguchi, M., Yanagida, T., 2000. Phys. Rev. Lett. 85, 3572.

Kolb, E.W., Turner, M.S., 1990. The Early Universe. (Addison-Wesley, Reading).

Peebles, P.J.E., Ratra, B., 1988. Astrophys. J. Lett. 325, L17.

Perlmutter, S. et al., 1999. Astrophys. J. 517, 565.

Riess, A.G. et al., 1998. Astron. J. 116, 1009.

Wang, L., Caldwell, R.R., Ostriker, J.P., and Steinhardt, P.J., 2000. Astrophis. J. $530,17$.

Weinberg, S., 1987. Phys. Rev. Lett. 61, 1.

Weinberg, S., 1989. Rev. Mod. Phys. 61, 1.

Zeldovich, Ya.B., Novikov, I.D., 1983. The Structure and Evolution of the Universe. (The Univ. Chicago Press, Chicago and London).

Zlatev, I., Wang, L., Steinhard, P.J., 1999. Phys. Rev. Lett. 82, 896. 